\title{
Long Range Forces From Pseudoscalar Exchange
}

\author{
F. Ferrer and J. A. Grifols \\ Grup de Física Teòrica and Institut de Física d'Altes Energies, \\ Universitat Autònoma de Barcelona, E-08193 Bellaterra, Barcelona, Spain
}

(May 1998)

\begin{abstract}
Using dispersion theoretic techniques, we consider coherent long range forces arising from double pseudoscalar exchange among fermions. We find that Yukawa type coupling leads to $1 / r^{3}$ spin independent attractive potentials whereas derivative coupling renders $1 / r^{5}$ spin independent repulsive potentials.
\end{abstract}

Typeset using REVTEX 


\section{INTRODUCTION}

Many extensions of the Standard model predict the existence of light scalar particles. The axion may be the most debated one but there are also approximate Nambu-Goldstone fields associated with family symmetries, or moduli fields, or dilatons, or superpartners of the gravitino [1]. Exchange of such particles by ordinary matter will induce forces that extend over the Compton wavelength of the particle [2, 56,6]. However, the effect will be felt by bulk matter only if the potential is spin-independent so that forces can add up coherently over macroscopic distances. Now, a pseudoscalar particle, such as the axion, is coupled to fermions via a $\gamma_{5}$ which, in the nonrelativistic limit, flips the spin. Therefore, single pseudoscalar exchange leads to spin-dependent forces that do not extend over macroscopic unpolarized bodies [3,5]. A double exchange of pseudoscalars on the other hand can coherently sum over a macroscopic sample of matter because it can leave the spin unflipped. The explicit form of these forces has been derived and their phenomenological consequences explored in previous work in the context of nonrelativistic "old fashioned perturbation theory" [7]. Here we reopen the question of pseudoscalar mediated forces in the light of the powerful dispersion theoretical techniques devised by Feinberg and Sucher and collaborators that make extensive use of full relativistic quantum field theory [8,9].

In section $\llbracket$ we give the necessary theoretical background which is nothing but a brief resumé of the seminal work by Feinberg and Sucher. Section III is devoted to the Yukawa type interaction and section [V] deals with derivatively coupled scalars. We shall see that the two interactions produce quite different potentials [10], a fact that could not have been

derived in a purely non-relativistic framework [11]. The paper ends with a brief summary and conclusions contained in section $\mathrm{\square}$. 


\section{DISPERSIVE FORCES}

Following the general strategy devised by Feinberg and Sucher [8,9], we define a potential in a given Quantum Field Theory by equating the scattering amplitude for a two body process that follows from the usual Feynman rules, with the transition amplitude associated to a Schrödinger type equation solved à la Lippmann-Schwinger. Let us be explicit and consider elastic scattering of particles $\mathrm{A}$ and $\mathrm{B}$ with four momenta $p_{a}$ and $p_{b}$ in the initial state and $p_{a}^{\prime}$ and $p_{b}^{\prime}$ in the final state. The Mandelstam variables are then:

$$
s=\left(p_{a}+p_{b}\right)^{2} \quad t=Q^{2} \quad u=\left(p_{a}-p_{b}^{\prime}\right)^{2}
$$

with $Q=p_{a}-p_{a}^{\prime}=-p_{b}+p_{b}^{\prime}$.

In the C.M. we write the momenta as

$$
\begin{gathered}
p_{a}=\left(E_{a}, \mathbf{p}\right) \quad p_{b}=\left(E_{b},-\mathbf{p}\right) \\
p_{a}^{\prime}=\left(E_{a}, \mathbf{p}^{\prime}\right) \quad p_{b}^{\prime}=\left(E_{b},-\mathbf{p}^{\prime}\right) .
\end{gathered}
$$

Now $s=W^{2}$ where $W=E_{a}+E_{b}$ and $t=-\mathbf{Q}^{2}$ with $Q=(0, \mathbf{Q})$, whose physical region is

$$
s \geq s_{0} \quad \text { and } \quad-4 \mathbf{p}^{2} \leq t \leq 0
$$

where

$$
s_{0}=\left(m_{a}+m_{b}\right)^{2} \quad \mathbf{p}^{2}=\left[s-\left(m_{a}+m_{b}\right)^{2}\right]\left[s-\left(m_{a}-m_{b}\right)^{2}\right] / 4 s .
$$

The transition from initial state $i$ to final state $f$ is described in Quantum Field Theory by the transition matrix element

$$
T_{f i}=N_{f} \mathcal{M}_{f i} N_{i}
$$

where $N_{f, i}$ are normalization factors of one particle states and $\mathcal{M}_{f i}$ is the invariant Feynman amplitude.

The definition of our potential follows now from identifying this transition amplitude with 


$$
T_{f i}=\left\langle\mathbf{p}^{\prime},-\mathbf{p}^{\prime}\left|V+V\left(W-h_{0}-V+i \epsilon\right)^{-1} V\right| \mathbf{p},-\mathbf{p}\right\rangle \frac{m_{a} m_{b}}{E_{a} E_{b}}
$$

where $h_{0}$ is the sum of the free Dirac Hamiltonians for particles A and B. Here all quantities are referred to the C.M.

The Feynman amplitude $\mathcal{M}$ is understood as a series expansion in (even) powers of the coupling constant associated to single, double, ... particle exchange in the t-channel. We assume that the potential $\mathrm{V}$ also admits a series expansion

$$
V=V^{(2)}+V^{(4)}+\ldots
$$

So we determine $\mathrm{V}$ order by order in perturbation theory through

$$
\begin{aligned}
& \left\langle\mathbf{p}^{\prime},-\mathbf{p}^{\prime}\left|V^{(2)}\right| \mathbf{p},-\mathbf{p}\right\rangle=\mathcal{M}_{f i}^{(2)} \\
& \left\langle\mathbf{p}^{\prime},-\mathbf{p}^{\prime}\left|V^{(4)}\right| \mathbf{p},-\mathbf{p}\right\rangle=\mathcal{M}_{f i}^{(4)}-\left\langle\mathbf{p}^{\prime},-\mathbf{p}^{\prime}\left|V^{(2)}\left(W-h_{0}+i \epsilon\right)^{-1} V^{(2)}\right| \mathbf{p},-\mathbf{p}\right\rangle .
\end{aligned}
$$

Here we should point out a technicality. The potential we are after must have the form $[12$

$$
V=\Lambda_{++} U \Lambda_{++}
$$

where $\Lambda_{++}=\Lambda_{+; a} \Lambda_{+; b}$ is an operator that projects on the positive energy states of $h_{0}$. Because $\Lambda_{++}|\mathbf{p},-\mathbf{p}\rangle=|\mathbf{p},-\mathbf{p}\rangle$ and $\Lambda_{++}^{2}=\Lambda_{++}$we can rewrite the previous equations that determine the potential as,

$$
\begin{aligned}
& \left\langle\mathbf{p}^{\prime},-\mathbf{p}^{\prime}\left|U^{(2)}\right| \mathbf{p},-\mathbf{p}\right\rangle=\mathcal{M}_{f i}^{(2)} \\
& \left\langle\mathbf{p}^{\prime},-\mathbf{p}^{\prime}\left|U^{(4)}\right| \mathbf{p},-\mathbf{p}\right\rangle=\mathcal{M}_{f i}^{(4)}-\left\langle\mathbf{p}^{\prime},-\mathbf{p}^{\prime}\left|U^{(2)}\left(W-h_{0}+i \epsilon\right)^{-1} \Lambda_{++} U^{(2)}\right| \mathbf{p},-\mathbf{p}\right\rangle
\end{aligned}
$$

In principle the above equations permit an iterative determination of the potential to the desired order. However we are not done yet because we would like to have our potential in position space and what we have is the operator $U$ in the momentum representation. Therefore, we should Fourier transform our results back to configuration space, i.e. we wish to find $U^{(n)}(r)$ such that 


$$
\left\langle\mathbf{p}^{\prime},-\mathbf{p}^{\prime}\left|U^{(n)}\right| \mathbf{p},-\mathbf{p}\right\rangle=\int d \mathbf{r} e^{i \mathbf{Q} \cdot \mathbf{r}} U^{(n)}(r)
$$

Inversion of the above equation, however, requires knowing the function $\mathcal{M}$ for all values of three momentum. But we only know the scattering amplitude on shell, i.e. for $\mathbf{p}^{2}=\mathbf{p}^{\prime 2}$. We can use the fact that $\mathcal{M}(s, t)$ is an analytic function of $\mathrm{t}$ and so analytically extend its domain beyond the physical region, i.e. for all values of $\mathrm{Q}^{2}=t$.

Suppose $\mathcal{M}(s, t)$ is analytic everywhere except for branch cuts on the real axis and furthermore, it vanishes for large $|t|$. Then, using Cauchy's theorem, we can write

$$
\begin{aligned}
& \mathcal{M}^{(R)}=\frac{1}{\pi} \int_{t_{0}}^{\infty} d t^{\prime} \frac{\rho^{(R)}\left(s, t^{\prime}\right)}{t^{\prime}-t} \\
& \mathcal{M}^{(L)}=\frac{1}{\pi} \int_{-\infty}^{\bar{t}_{0}} d t^{\prime} \frac{\rho^{(L)}\left(s, t^{\prime}\right)}{t^{\prime}-t}
\end{aligned}
$$

where $\rho(s, t)=\frac{[\mathcal{M}]_{t}}{2 i}$ is the spectral density and $[\mathcal{M}]_{t}$ is the discontinuity of $\mathcal{M}$ across the cut. Only the piece of the amplitude arising from the right hand cut will be of interest to us for only this piece leads to a long range potential [13]. Assuming that the basic relations eqs (11) and (12) hold also in the extended domain, we can Fourier invert them as follows!,

$$
U^{(n)}(r ; s)=\frac{1}{(2 \pi)^{3}} \int d \mathbf{Q} e^{-\mathbf{Q} \cdot \mathbf{r}} \mathcal{M}^{(n)}\left(s,-\mathbf{Q}^{2}\right)
$$

We use now the spectral representation given before to obtain

$$
U^{(n)}(r ; s)=\frac{1}{4 \pi^{2} r} \int_{t_{0}}^{\infty} d t \rho^{(n)}(s, t) e^{-\sqrt{t} r}
$$

where, to reach this final form, we conveniently changed the order in which integrals were done. In short, obtaining long range potentials amounts to calculating t-channel discontinuities in Feynman diagrams and performing a Laplace transform. We shall see how things work out in detail as we do our specific calculations in the next two sections.

\footnotetext{
${ }^{1}$ Notice that our generalized potential will depend on the parameter $s$.
} 


\section{THE YUKAWA COUPLING}

Our starting point is the Lagrangian density,

$$
\mathcal{L}_{\text {int }}^{Y}=-i g \bar{\Psi}(x) \gamma^{5} \Psi(x) \Phi(x)
$$

where $\psi$ is a fermion field and $\phi$ is the pseudoscalar field which we take to be massless 2 . The potential associated to single particle exchange is easily obtained from the discontinuity associated to the diagram in Fig. 1. The spectral density function $\rho^{(2)}(s, t)$ is in this case

$$
\rho^{(2)}(s, t)=\pi g^{2} \bar{u}\left(p_{a}^{\prime}\right) \gamma^{5} u\left(p_{a}\right) \bar{u}\left(p_{b}^{\prime}\right) \gamma^{5} u\left(p_{b}\right) \delta(t)
$$

After Laplace transforming we get the relativistic potential operator

$$
U^{(2)}=\frac{g^{2}}{4 \pi r} \gamma_{a}^{0} \gamma_{a}^{5} \gamma_{b}^{0} \gamma_{b}^{5}
$$

where subindices make explicit that Dirac matrices act either on spinor A or spinor B.

The nonrelativistic limit of equation above leads to the well known spin-dependent potential

$$
V_{n r}^{(2)}=\frac{g^{2}}{4 \pi r\left(2 m_{a}\right)\left(2 m_{b}\right)} \boldsymbol{\sigma}_{a} \cdot \boldsymbol{\nabla} \otimes \boldsymbol{\sigma}_{b} \cdot \boldsymbol{\nabla}
$$

with $m_{a}$ and $m_{b}$, the masses of particles A and B respectively. What we are really interested in is $U^{(4)}$, i.e. the potential due to two-particle exchange. To this end we need the discontinuities of diagrams in Fig. 2 and the discontinuity of the subtraction term in eq (12) (iteration of the lowest order potential $\left.U^{(2)}\right)$.

The Feynman amplitude associated to Fig. 2 can be written

$$
\begin{array}{r}
\mathcal{M}^{(4)}=\frac{i}{2 !(2 \pi)^{4}} \int d^{4} k d^{4} k^{\prime} \delta^{(4)}\left(Q-k-k^{\prime}\right) \frac{1}{k^{2}+i \epsilon} \frac{1}{{k^{\prime}}^{2}+i \epsilon} \\
\mathcal{M}_{a}^{C}\left(-k, k^{\prime} ; P_{a}\right) \mathcal{M}_{b}^{C}\left(k,-k^{\prime} ; P_{b}\right)
\end{array}
$$

\footnotetext{
${ }^{2}$ For scalars with mass, the long distance potentials are damped with Yukawa exponentials. In this case, our results are valid for distances on the order or smaller than the Compton wavelength of the exchanged particles.
} 
in terms of the Compton amplitude, depicted in Fig. 3,

$$
\mathcal{M}^{C}\left(k, k^{\prime} ; P\right)=g^{2} \bar{u}\left(p^{\prime}\right)\left[\frac{\not k}{2 p \cdot k}+\frac{\not k^{\prime}}{2 p \cdot k^{\prime}}\right] u(p) .
$$

Making use of the Dirac equation and trading the propagators for their discontinuities, i.e.

$$
\frac{1}{k^{2}+i \epsilon} \longrightarrow-2 \pi i \delta\left(k^{2}\right) \Theta\left(k^{0}\right)
$$

we arrive at

$$
\left[\mathcal{M}^{(4)}\right]_{t}=-\frac{i g^{4}}{8 \pi^{2}} \int d \Phi \bar{u}_{a}^{\prime}\left[\frac{p_{a} \cdot\left(k^{\prime}-k\right) \not k}{2 p_{a} \cdot k^{\prime} p_{a} \cdot k}\right] u_{a} \bar{u}_{b}^{\prime}\left[\frac{p_{b} \cdot\left(k^{\prime}-k\right) \not k}{2 p_{b} \cdot k^{\prime} p_{b} \cdot k}\right] u_{b}
$$

with the two particle phase space explicitly given by

$$
d \Phi=\delta\left(Q-k-k^{\prime}\right) \delta\left(k^{2}\right) \delta\left(k^{2}\right) \Theta\left(k^{0}\right) \Theta\left(k^{\prime 0}\right) d^{4} k d^{4} k^{\prime}
$$

It is convenient to do the integrals in the C.M. of the pseudoscalars, i.e. to go to the t-channel, and then use crossing symmetry to recover the original amplitude. We follow here the notation in reference [14] where they deal with a related problem. First define momenta as,

$$
\begin{array}{cc}
p_{a}=\left(\frac{\sqrt{t}}{2}, \mathbf{p}\right) & p_{\bar{a}}=-p_{a}^{\prime}=\left(\frac{\sqrt{t}}{2},-\mathbf{p}\right) \\
p_{\bar{b}}=-p_{b}=\left(\frac{\sqrt{t}}{2},-\mathbf{p}^{\prime}\right) & p_{b}^{\prime}=\left(\frac{\sqrt{t}}{2}, \mathbf{p}^{\prime}\right) \\
k=\left(\frac{\sqrt{t}}{2}, \mathbf{k}\right) & k^{\prime}=\left(\frac{\sqrt{t}}{2},-\mathbf{k}\right)
\end{array}
$$

introduce next the unit imaginary vectors

$$
\begin{aligned}
\mathbf{p} & =i \xi_{a} m_{a} \hat{\mathbf{p}} \\
\mathbf{p}^{\prime} & =i \xi_{b} m_{b} \hat{\mathbf{p}}^{\prime}
\end{aligned}
$$

with $\xi_{a, b} \equiv \sqrt{1-\frac{t}{4 m_{a, b}^{2}}}$ and $\hat{\mathbf{p}}, \hat{\mathbf{p}}^{\prime}$ are unitary complex vectors verifying $\hat{\mathbf{p}} \cdot \hat{\mathbf{p}}=-1$ so that all particles are on-shell.

Now the discontinuity can be put in the form 


$$
\left[\mathcal{M}^{(4)}\right]_{t}=\frac{i g^{4}}{4 \pi b t} \int \frac{d \Omega}{4 \pi} \frac{x_{a} x_{b}}{d_{a} d_{b}} \quad \bar{u}_{a}^{\prime} \not k u_{a} \bar{u}_{b}^{\prime} \not k u_{b}
$$

where, to simplify expressions, we use

$$
\begin{gathered}
b \equiv m_{a} \xi_{a} m_{b} \xi_{b} \\
x_{a} \equiv \hat{\mathbf{p}} \cdot \hat{k} \quad x_{b} \equiv \hat{\mathbf{p}}^{\prime} \cdot \hat{k} \\
d_{a, b} \equiv \tau_{a, b}^{2}+x_{a, b}^{2} \\
\tau_{a, b} \equiv \frac{\sqrt{t}}{2 \xi_{a, b} m_{a, b}}
\end{gathered}
$$

The integration to be carried out is an angular average. We use the shorthand: $\int \frac{d \Omega}{4 \pi} f \equiv$ $\langle f\rangle$. Hence, the discontinuity takes the form

$$
\left[\mathcal{M}^{(4)}\right]_{t}=\frac{i g^{4}}{4 \pi b t} \bar{u}_{a}^{\prime} \gamma_{\mu} u_{a} \bar{u}_{b}^{\prime} \gamma_{\nu} u_{b} \mathcal{T}^{\mu \nu}
$$

with $\mathcal{T}^{\mu \nu} \equiv\left\langle\frac{x_{a} x_{b}}{d_{a} d_{b}} k^{\mu} k^{\nu}\right\rangle$

Lorentz covariance dictates the following decomposition

$$
\begin{aligned}
\mathcal{T}^{\mu \nu}= & a_{1} P_{a}^{\mu} P_{a}^{\nu}+a_{2} P_{b}^{\mu} P_{b}^{\nu}+a_{3}\left(P_{a}^{\mu} P_{b}^{\nu}+P_{b}^{\mu} P_{a}^{\nu}\right)+a_{4} g^{\mu \nu} \\
& +a_{5} Q^{\mu} Q^{\nu}+a_{6}\left(Q^{\mu} P_{a}^{\nu}+P_{a}^{\mu} Q^{\nu}\right)+a_{7}\left(Q^{\mu} P_{b}^{\nu}+P_{b}^{\mu} Q^{\nu}\right)
\end{aligned}
$$

in terms of the three independent momenta

$$
P_{a} \equiv p_{a}+p_{a}^{\prime} \quad P_{b} \equiv p_{b}+p_{b}^{\prime} \quad Q \equiv k+k^{\prime}
$$

The coefficients $a_{i}$ can be found to be combinations of scalar integrals as shown in the appendix. Now, in the C.M. of the incident particles, the relations

$$
\begin{aligned}
\bar{u}_{a}^{\prime} P_{a} u_{a} \bar{u}_{b}^{\prime} P_{a} u_{b} & =4 m_{a} \bar{u}_{a}^{\prime} u_{a} \bar{u}_{b}^{\prime}\left(W \gamma_{0}-m_{b}\right) u_{b} \\
\bar{u}_{a}^{\prime} P_{b} u_{a} \bar{u}_{b}^{\prime} P_{b} u_{b} & =4 m_{b} \bar{u}_{a}^{\prime}\left(W \gamma_{0}-m_{a}\right) u_{a} \bar{u}_{b}^{\prime} u_{b} \\
\bar{u}_{a}^{\prime} P_{a} u_{a} \bar{u}_{b}^{\prime} P_{b} u_{b} & =4 m_{a} m_{b} \bar{u}_{a}^{\prime} u_{a} \bar{u}_{b}^{\prime} u_{b} \\
\bar{u}_{a}^{\prime} P_{b} u_{a} \bar{u}_{b}^{\prime} P_{a} u_{b} & =4 \bar{u}_{a}^{\prime}\left(W \gamma_{0}-m_{a}\right) u_{a} \bar{u}_{b}^{\prime}\left(W \gamma_{0}-m_{b}\right) u_{b} \\
\bar{u}_{a}^{\prime} Q u_{a} & =0 \\
\bar{u}_{b}^{\prime} Q u_{b} & =0
\end{aligned}
$$


are easily established with the help of the Dirac equation. This leads directly to

$$
\begin{aligned}
{\left[\mathcal{M}^{(4)}\right]_{t} } & =\frac{i g^{4}}{4 \pi b t} \bar{u}_{a}^{\prime} \bar{u}_{b}^{\prime}\left[4 m_{a} m_{b}\left(2 a_{3}-a_{1}-a_{2}\right)+4 m_{b} W \gamma_{a}^{0}\left(a_{2}-a_{3}\right)\right. \\
& \left.+4 m_{a} W \gamma_{b}^{0}\left(a_{1}-a_{3}\right)+\gamma_{a}^{0} \gamma_{b}^{0}\left(4 W^{2} a_{3}+a_{4}\right)-\gamma_{a} \gamma_{b} a_{4}\right] u_{a} u_{b}
\end{aligned}
$$

This discontinuity is a complex function since the $a_{i}$ are complex and hence adds an imaginary component to the spectral density which would finally contribute an imaginary piece to the potential. Inspection of equation (36) immediately tells us that the offending piece comes from the imaginary parts of the $a_{i}$. But we should recall that we still have to subtract the contribution from the iterated lowest order potential. It turns out that its imaginary part exactly cancels the unwanted contribution coming from equation (36). Indeed we have explicitly checked this to be the case. However, in order to make this paper not too lengthy, we do not include the intermediate steps of the calculation. We only report on the result, i.e.

$$
\begin{aligned}
\Re\left[\mathcal{M}^{(4)}\right]_{t}= & \frac{g^{4}}{8 p W} \bar{u}_{a}^{\prime} \bar{u}_{b}^{\prime}\left[\frac{t-4 p^{2}}{\left(4 p^{2}+t\right)^{2}}\left(E_{a} \gamma_{a}^{0}-m_{a}\right)\left(E_{b} \gamma_{b}^{0}-m_{b}\right)\right. \\
& \left.-\frac{p^{2}}{4 p^{2}+t} \gamma_{a} \cdot \gamma_{b}\right] u_{a} u_{b} .
\end{aligned}
$$

The relevant contribution to the long range potential comes from the real parts of the $a_{i}$, that is the imaginary part of (36), once the contribution of the iterated potential has been subtracted. Let us elaborate on the iteration amplitude,

$$
\mathcal{M}_{I}=\left\langle\mathbf{p}^{\prime},-\mathbf{p}^{\prime}\left|U^{(2)}\left(W-h_{0}+i \epsilon\right)^{-1} \Lambda_{++} U^{(2)}\right| \mathbf{p},-\mathbf{p}\right\rangle
$$

This formal expression can be recast in the explicit form?

$$
\begin{aligned}
\mathcal{M}_{I}= & \frac{g^{4}}{8 \pi^{2}} \int l^{2} d l \int \frac{d \Omega}{4 \pi} u_{a}^{\dagger \prime} u_{b}^{\dagger \prime} \gamma_{a}^{0}\left(E_{a}^{\prime} \gamma_{a}^{0}-\gamma_{a} \cdot \mathbf{l}-m_{a}\right) \\
& \gamma_{b}^{0}\left(E_{b}^{\prime} \gamma_{b}^{0}+\gamma_{b} \cdot \mathbf{l}-m_{b}\right) u_{a} u_{b} \mathcal{C}(p, l) \frac{1}{\mathbf{q}^{\prime 2}} \frac{1}{\mathbf{q}^{2}}
\end{aligned}
$$

\footnotetext{
${ }^{3}$ This integral as it stands is infrared divergent. A fictitious mass regulator is understood to be introduced in the scalar propagators which is set to zero after the integrations are performed.
} 
with $\mathbf{q} \equiv \mathbf{p}-\mathbf{l}$ and $\mathbf{q}^{\prime} \equiv \mathbf{p}^{\prime}-\mathbf{l}$ and $\mathcal{C}(p, l) \equiv \frac{1}{E_{a}^{\prime} E_{b}^{\prime}\left(W-W^{\prime}+i \epsilon\right)}$.

The integration over momentum 1 reflects the fact that we have inserted a complete set of plane wave intermediate states in (38). We have also used

$$
\Lambda_{+; a}(\mathbf{l})=\frac{E_{a}^{\prime}+\gamma_{a}^{0} \gamma_{a} \cdot \mathbf{l}+\gamma_{a}^{0} m_{a}}{2 E_{a}^{\prime}}
$$

where $E_{a}^{\prime}=\sqrt{m_{a}^{2}+l^{2}}$.

The iteration amplitude can be conveniently put as follows

$$
\begin{aligned}
\mathcal{M}_{I}= & \frac{g^{4}}{8 \pi^{2}} \int l^{2} d l \bar{u}_{a}^{\prime} \bar{u}_{b}^{\prime}\left\{\left(E_{a}^{\prime} \gamma_{a}^{0}-m_{a}\right)\left(E_{b}^{\prime} \gamma_{b}^{0}-m_{b}\right) \mathcal{L}-\gamma_{a} \cdot \mathcal{V}\left(E_{b}^{\prime} \gamma_{b}^{0}-m_{b}\right)\right. \\
& \left.+\left(E_{a}^{\prime} \gamma_{a}^{0}-m_{a}\right) \gamma_{b} \cdot \mathcal{V}-\gamma_{a}^{i} \gamma_{b}^{j} \Upsilon_{i j}\right\} u_{a} u_{b} \mathcal{C}(p, l)
\end{aligned}
$$

where

$$
\begin{aligned}
\mathcal{L} & \equiv \int \frac{d \Omega}{4 \pi} \frac{1}{\mathbf{q}^{\prime 2}} \frac{1}{\mathbf{q}^{2}} \\
\mathcal{V} & \equiv \int \frac{d \Omega}{4 \pi} \mathbf{l} \frac{1}{\mathbf{q}^{\prime 2}} \frac{1}{\mathbf{q}^{2}} \\
\Upsilon_{i j} & \equiv \int \frac{d \Omega}{4 \pi} l_{i} l_{j} \frac{1}{\mathbf{q}^{\prime 2}} \frac{1}{\mathbf{q}^{2}}
\end{aligned}
$$

and they can be found in the appendix.

A little bit of Dirac algebra and the results in the appendix allow us to write the discontinuity of $\mathcal{M}_{I}$ as

$$
\begin{aligned}
{\left[\mathcal{M}_{I}\right]_{t}=} & \frac{g^{4}}{8 \pi^{2}} \int l^{2} d l \bar{u}_{a}^{\prime} \bar{u}_{b}^{\prime}\left\{\left(E_{a}^{\prime} \gamma_{a}^{0}-m_{a}\right)\left(E_{b}^{\prime} \gamma_{b}^{0}-m_{b}\right)\right. \\
& -2 \frac{p^{2}+l^{2}}{4 p^{2}+t}\left[\left(E_{a} \gamma_{a}^{0}-m_{a}\right)\left(E_{b}^{\prime} \gamma_{b}^{0}-m_{b}\right)+\left(E_{a}^{\prime} \gamma_{a}^{0}-m_{a}\right)\left(E_{b} \gamma_{b}^{0}-m_{b}\right)\right] \\
& +\frac{4}{4 p^{2}+t}\left(2 \frac{\left(p^{2}+l^{2}\right)^{2}}{4 p^{2}+t}-l^{2}\right)\left(E_{a} \gamma_{a}^{0}-m_{a}\right)\left(E_{b} \gamma_{b}^{0}-m_{b}\right) \\
& \left.+\gamma_{a} \cdot \gamma_{b}\left(\frac{\left(p^{2}+l^{2}\right)^{2}}{4 p^{2}+t}-l^{2}\right)\right\}[\mathcal{L}]_{t} u_{a} u_{b} \mathcal{C}(p, l) .
\end{aligned}
$$

The explicit form for $[\mathcal{L}]_{t}$ is given in the appendix. Note that in $(45)$ we have

$$
\frac{1}{W-W^{\prime}+i \epsilon}=\wp\left(\frac{1}{W-W^{\prime}}\right)-i \pi \delta\left(W-W^{\prime}\right) \text {. }
$$


The Dirac delta piece gives a contribution that, as already advertised, will exactly cancel the real part of the fourth order discontinuity function (37). The principal part integral can be cast in the form

$$
i \Im\left[\mathcal{M}_{I}\right]_{t}=\frac{i g^{4}}{16 \pi \sqrt{t}\left(4 p^{2}+t\right)^{2}} \wp \int_{-1}^{1} \frac{d x}{\sqrt{1-x^{2}}} \mathcal{C}(p, l) \bar{u}_{a}^{\prime} \bar{u}_{b}^{\prime} \mathcal{N} u_{a} u_{b}
$$

where we changed the integration variable via the relation

$$
l^{2}=\frac{a^{\prime}+b^{\prime} x}{2} \equiv \frac{1}{2}\left[\left(2 p^{2}+t\right)+\sqrt{t\left(4 p^{2}+t\right)} x\right]
$$

and we used the shorthand

$$
\begin{aligned}
\mathcal{N} \equiv & \left(4 p^{2}+t\right)^{2}\left(E_{a}^{\prime} \gamma_{a}^{0}-m_{a}\right)\left(E_{b}^{\prime} \gamma_{b}^{0}-m_{b}\right)-2\left(4 p^{2}+t\right)\left(p^{2}+l^{2}\right) \\
& {\left[\left(E_{a} \gamma_{a}^{0}-m_{a}\right)\left(E_{b}^{\prime} \gamma_{b}^{0}-m_{b}\right)+\left(E_{a}^{\prime} \gamma_{a}^{0}-m_{a}\right)\left(E_{b} \gamma_{b}^{0}-m_{b}\right)\right] } \\
& +4\left(2\left(p^{2}+l^{2}\right)^{2}-l^{2}\left(4 p^{2}+t\right)\right)\left(E_{a} \gamma_{a}^{0}-m_{a}\right)\left(E_{b} \gamma_{b}^{0}-m_{b}\right) \\
& -\gamma_{a} \cdot \gamma_{b}\left(4 p^{2}+t\right)\left(\left(4 p^{2}+t\right) l^{2}-\left(p^{2}+l^{2}\right)^{2}\right) .
\end{aligned}
$$

It is convenient now to split the function $\mathcal{C}(p, l)$ as

$$
\begin{aligned}
\mathcal{C}(p, l) & \equiv \mathcal{C}_{1}(p, l)+\mathcal{C}_{2}(p, l) \\
& \equiv \frac{2}{W\left(p^{2}-l^{2}\right)}+\frac{1}{E_{a}^{\prime} E_{b}^{\prime} W}\left(\frac{p^{2}+l^{2}+m_{a}^{2}+m_{b}^{2}}{E_{a} E_{b}+E_{a}^{\prime} E_{b}^{\prime}}+\frac{W^{\prime}}{W+W^{\prime}}\right) .
\end{aligned}
$$

The integral above cannot be done exactly and we will expand the integral in a power series in $t$ and $p^{2}$. This is a licit procedure because we will perform a Laplace transform that heavily weighs the small $\mathrm{t}$ region of the spectral function when determining the long range (large r) potential and, we will eventually take the non relativistic limit of the potential, i.e. for $p^{2} \sim 0$. Furthermore, each extra power of $t$ or $p^{2}$ implies a correction to the potential with an extra power of $r^{-1}$. We see from equation (50) that the calculation will involve doing integrals of the type

$$
I_{c 1}(n) \equiv \int_{-1}^{1} \frac{d x}{\sqrt{1-x^{2}}} \frac{l^{n}}{p^{2}-l^{2}}
$$




$$
I_{c 2}(n) \equiv \int_{-1}^{1} \frac{d x}{\sqrt{1-x^{2}}} l^{n} .
$$

The explicit results of the integrals needed in our calculation are also given in the appendix. Armed with all this artillery we find for the $\mathcal{C}_{1}$ piece of the discontinuity $\left[\mathcal{M}_{I}\right]_{t}$ :

$$
\begin{aligned}
i \Im\left[\mathcal{M}_{I}^{C_{1}}\right]_{t}= & \frac{i g^{4}}{8 \sqrt{t}\left(4 p^{2}+t\right)^{2}\left(m_{a}+m_{b}\right)} \bar{u}_{a}^{\prime} \bar{u}_{b}^{\prime} \\
& \left\{m_{a} m_{b}\left[4 t-\frac{2 t p^{2}}{m_{a} m_{b}}+\frac{p^{4} t}{2 m_{a} m_{b}}\left(\frac{1}{m_{a}^{2}}+\frac{1}{m_{b}^{2}}+\frac{1}{m_{a} m_{b}}\right)\right]\right. \\
+\gamma_{a}^{0} m_{a} m_{b}\left[-4 t+\frac{2\left(m_{a}-m_{b}\right)}{m_{a}^{2} m_{b}} t p^{2}\right. & \\
+ & \left.\frac{t^{3}}{32 m_{a}^{4}}+\frac{t^{2} p^{2}}{4 m_{a}^{4}}+\frac{-m_{a}^{3}-m_{a}^{2} m_{b}+m_{a} m_{b}^{2}+2 m_{b}^{3}}{2 m_{a}^{4} m_{b}^{3}} t p^{4}\right] \\
+\gamma_{b}^{0} \quad m_{a} m_{b}\left[-4 t+\frac{2\left(m_{b}-m_{a}\right)}{m_{a} m_{b}^{2}} t p^{2}\right. & \\
+ & \left.\frac{t^{3}}{32 m_{b}^{4}}+\frac{t^{2} p^{2}}{4 m_{b}^{4}}+\frac{2 m_{a}^{3}+m_{a}^{2} m_{b}-m_{a} m_{b}^{2}-m_{b}^{3}}{2 m_{a}^{3} m_{b}^{4}} t\right] \\
+\gamma_{a}^{0} \gamma_{b}^{0} & m_{a} m_{b}\left[4 t+\left(\frac{2}{m_{a}^{2}}+\frac{2}{m_{b}^{2}}-\frac{2}{m_{a} m_{b}}\right) t p^{2}\right. \\
& -t^{3}\left(\frac{1}{32 m_{a}^{4}}+\frac{1}{32 m_{b}^{4}}+\frac{1}{8 m_{a}^{2} m_{b}^{2}}\right)-t^{2} p^{2}\left(\frac{1}{4 m_{a}^{4}}+\frac{1}{4 m_{b}^{4}}+\frac{1}{m_{a}^{2} m_{b}^{2}}\right) \\
& -t p^{4} \frac{2 m_{a}^{4}+m_{a}^{3} m_{b}+m_{a}^{2} m_{b}^{2}+m_{a} m_{b}^{3}+2 m_{b}^{4}}{2 m_{a}^{4} m_{b}^{4}} \\
+\gamma_{a} \cdot \gamma_{b} & {\left.\left[2 t p^{2}+\frac{t^{2}}{2}-\frac{t^{2} p^{2}}{4 m_{a} m_{b}}-\frac{t p^{4}}{m_{a} m_{b}}\right]\right\} u_{a} u_{b} }
\end{aligned}
$$

and for the $\mathcal{C}_{2}$ piece:

$$
\begin{aligned}
i \Im\left[\mathcal{M}_{I}^{C_{2}}\right]_{t} & =\frac{i g^{4}}{16 \sqrt{t}\left(4 p^{2}+t\right)^{2}\left(m_{a}+m_{b}\right) m_{a} m_{b}} \bar{u}_{a}^{\prime} \bar{u}_{b}^{\prime}\{ \\
& \gamma_{a}^{0} \quad m_{a} m_{b}\left[\frac{m_{a}^{2}+m_{a} m_{b}+m_{b}^{2}}{16 m_{a}^{3} m_{b}} t\left(4 p^{2}+t\right)^{2}\right] \\
& +\gamma_{b}^{0} \quad m_{a} m_{b}\left[\frac{m_{a}^{2}+m_{a} m_{b}+m_{b}^{2}}{16 m_{a} m_{b}^{3}} t\left(4 p^{2}+t\right)^{2}\right] \\
& -\gamma_{a}^{0} \gamma_{b}^{0}\left[\frac{m_{a}^{4}+m_{a}^{3} m_{b}+2 m_{a}^{2} m_{b}^{2}+m_{a} m_{b}^{3}+m_{b}^{4}}{16 m_{a}^{2} m_{b}^{2}} t\left(4 p^{2}+t\right)^{2}\right] \\
- & \left.\gamma_{a} \cdot \gamma_{b}\left[\frac{m_{a}^{2}+m_{a} m_{b}+m_{b}^{2}}{16 m_{a} m_{b}} t\left(4 p^{2}+t\right)^{2}\right]\right\} u_{a} u_{b} .
\end{aligned}
$$

In both equations above we kept only up to the powers of $t$ and $p^{2}$ that will be needed, either in this section or in the next section, to obtain the leading two-particle exchange 
potential. In this respect equation (54) does not contribute to the leading potential just under scrutiny. To obtain the spectral density $\rho^{(4)}$, we must finally perform the subtraction

$$
\rho^{(4)}(s, t)=\frac{\Im\left[\mathcal{M}^{(4)}\right]_{t}-\Im\left[\mathcal{M}_{I}\right]_{t}}{2}
$$

Recall that what enters $\rho^{(4)}$ is the real part of the $a_{i}$ in (36). Although the integrals that go in the $a_{i}$ are exactly given in the appendix, the required subtraction (55) and final Laplace transformation (17) demand that we here also expand the integrands in a power series in $t$ and $p^{2}$. After some lengthy algebra, we arrive at the final form for the imaginary part of $[\mathcal{M}]_{t}$

$$
\Im[\mathcal{M}]_{t}=\Im[\mathcal{M}]_{t}^{\text {odd }}+\Im[\mathcal{M}]_{t}^{\text {even }}
$$

where

$$
\begin{aligned}
& \Im\left[\mathcal{M}^{(4)}\right]_{t}^{\text {odd }}=\frac{g^{4} m_{a} m_{b}}{4\left(m_{a}+m_{b}\right) \sqrt{t}\left(4 p^{2}+t\right)^{2}} \bar{u}_{a}^{\prime} \bar{u}_{b}^{\prime}\{ \\
& {\left[2 t-\frac{t p^{2}}{m_{a} m_{b}}+\frac{-3 m_{a}^{4}+2 m_{a}^{2} m_{b}^{2}-3 m_{b}^{4}}{4 m_{a}^{4} m_{b}^{4}} t p^{4}\right.} \\
& +\frac{-3 m_{a}^{4}-m_{a}^{3} m_{b}+m_{a}^{2} m_{b}^{2}-m_{a} m_{b}^{3}-3 m_{b}^{4}}{8 m_{a}^{4} m_{b}^{4}} t^{2} p^{2} \\
& \left.+\frac{-3 m_{a}^{4}-m_{a}^{3} m_{b}+m_{a}^{2} m_{b}^{2}-m_{a} m_{b}^{3}-3 m_{b}^{4}}{64 m_{a}^{4} m_{b}^{4}} t^{3}\right] \\
& +\gamma_{a}^{0} \quad\left[-2 t+\frac{m_{a}-m_{b}}{m_{a}^{2} m_{b}} t p^{2}+\frac{3 m_{a}+4 m_{b}}{4 m_{a} m_{b}^{4}} t p^{4}\right. \\
& +\frac{3 m_{a}^{4}+5 m_{a}^{3} m_{b}+m_{a}^{2} m_{b}^{2}-m_{a} m_{b}^{3}-m_{b}^{4}}{8 m_{a}^{4} m_{b}^{4}} t^{2} p^{2} \\
& \left.+\frac{3 m_{a}^{4}+5 m_{a}^{3} m_{b}+m_{a}^{2} m_{b}^{2}-m_{a} m_{b}^{3}-m_{b}^{4}}{64 m_{a}^{4} m_{b}^{4}} t^{3}\right] \\
& +\gamma_{b}^{0} \quad\left[-2 t+\frac{m_{b}-m_{a}}{m_{a} m_{b}^{2}} t p^{2}+\frac{4 m_{a}+3 m_{b}}{4 m_{a}^{4} m_{b}} t p^{4}\right. \\
& +\frac{-m_{a}^{4}-m_{a}^{3} m_{b}+m_{a}^{2} m_{b}^{2}+5 m_{a} m_{b}^{3}+3 m_{b}^{4}}{8 m_{a}^{4} m_{b}^{4}} t^{2} p^{2} \\
& \left.+\frac{-m_{a}^{4}-m_{a}^{3} m_{b}+m_{a}^{2} m_{b}^{2}+5 m_{a} m_{b}^{3}+3 m_{b}^{4}}{64 m_{a}^{4} m_{b}^{4}} t^{3}\right] \\
& +\gamma_{a}^{0} \gamma_{b}^{0}\left[2 t+\frac{m_{a}^{2}-m_{a} m_{b}+m_{b}^{2}}{m_{a}^{2} m_{b}^{2}} t p^{2}+\frac{t p^{4}}{m_{a}^{2} m_{b}^{2}}\right.
\end{aligned}
$$




$$
\begin{aligned}
& +\frac{m_{a}^{4}+m_{a}^{3} m_{b}+m_{a}^{2} m_{b}^{2}+m_{a} m_{b}^{3}+m_{b}^{4}}{8 m_{a}^{4} m_{b}^{4}} t^{2} p^{2} \\
& \left.+\frac{m_{a}^{4}+m_{a}^{3} m_{b}+m_{a}^{2} m_{b}^{2}+m_{a} m_{b}^{3}+m_{b}^{4}}{64 m_{a}^{4} m_{b}^{4}} t^{3}\right] \\
+\gamma_{a} \cdot \gamma_{b}\left[\frac{p^{2} t}{m_{a} m_{b}}+\frac{t^{2}}{4 m_{a} m_{b}}+\frac{m_{a}^{2}+4 m_{a} m_{b}+m_{b}^{2}}{2 m_{a}^{3} m_{b}^{3}} t p^{4}\right. & \\
& \left.+\frac{3 m_{a}^{2}+5 m_{a} m_{b}+3 m_{b}^{2}}{8 m_{a}^{3} m_{b}^{3}} t^{2} p^{2}+\frac{2 m_{a}^{2}+m_{a} m_{b}+2 m_{b}^{2}}{32 m_{a}^{3} m_{b}^{3}} t^{3}\right] \\
\} & u_{a} u_{b}
\end{aligned}
$$

contains the odd powers of $\sqrt{t}$ and

$$
\begin{aligned}
\Im\left[\mathcal{M}^{(4)}\right]_{t}^{e v e n}= & \frac{g^{4}}{4 \pi} \bar{u}_{a}^{\prime} \bar{u}_{b}^{\prime}\left\{-\frac{\left(m_{a}+m_{b}\right) \gamma_{b}^{0}-m_{b}}{6 m_{a}^{2} m_{b}}-\frac{\left(m_{a}+m_{b}\right) \gamma_{a}^{0}-m_{a}}{6 m_{a} m_{b}^{2}}+\frac{\gamma_{a}^{\mu} \gamma_{\mu}^{b}}{4 m_{a} m_{b}}\right. \\
& \left.-\frac{m_{a} m_{b}+\left(\left(m_{a}+m_{b}\right) \gamma_{a}^{0}-m_{a}\right)\left(\left(m_{a}+m_{b}\right) \gamma_{b}^{0}-m_{b}\right)}{12 m_{a}^{2} m_{b}^{2}}\right\} u_{a} u_{b}
\end{aligned}
$$

contains the even powers of $\sqrt{t}$. We did this separation to emphasize that, after the subtraction in equation (55), only the term (58) survives to leading non-vanishing order. Indeed, equation (57) coincides exactly with equation (53) if we neglect terms beyond $t^{2}, p^{4}$, or $t p^{2}$.

The final step involves the Laplace transformation indicated by equation (17). Using the general formula

$$
\int_{0}^{\infty} t^{n} e^{-\sqrt{t} r} d t=\frac{2(2 n+1) !}{r^{2 n+2}}
$$

we get

$$
\begin{aligned}
U^{(4)}(r ; s)= & \frac{g^{4}}{16 \pi r^{3}} \bar{u}_{a}^{\prime} \bar{u}_{b}^{\prime}\left\{-\frac{\left(m_{a}+m_{b}\right) \gamma_{b}^{0}-m_{b}}{6 m_{a}^{2} m_{b}}-\frac{\left(m_{a}+m_{b}\right) \gamma_{a}^{0}-m_{a}}{6 m_{a} m_{b}^{2}}+\frac{\gamma_{a}^{\mu} \gamma_{\mu}^{b}}{4 m_{a} m_{b}}\right. \\
& \left.-\frac{m_{a} m_{b}+\left(\left(m_{a}+m_{b}\right) \gamma_{a}^{0}-m_{a}\right)\left(\left(m_{a}+m_{b}\right) \gamma_{b}^{0}-m_{b}\right)}{12 m_{a}^{2} m_{b}^{2}}\right\} u_{a} u_{b}
\end{aligned}
$$

which leads, in the non relativistic limit and concentrating only on the spin-independent terms of (60), to the long-range attractive potential:

$$
V_{n r}^{(4)}=-\frac{g^{4}}{64 \pi^{3} r^{3} m_{a} m_{b}} \mathbf{1}_{2}^{a} \otimes \mathbf{1}_{2}^{b}
$$

where this operator is supposed to act between two-component Pauli spinors. 


\section{THE DERIVATIVE COUPLING}

In this section we consider the interaction Lagrangian

$$
\mathcal{L}_{\text {int }}^{\text {der }}=\frac{g}{2 m} \bar{\Psi}(x) \gamma_{\mu} \gamma^{5} \Psi(x) \partial^{\mu} \Phi(x)
$$

which is how Goldstone bosons couple to fermions.

This derivative coupling leads to the same one particle exchange Feynman amplitude as before and therefore to the same lowest order potential (20). Hence the iteration amplitude will be also identical. However, the two particle exchange amplitude (see, Fig. 2) is different because the Compton amplitude that goes into (22) is different. Indeed, the Compton amplitude, corresponding to Fig. 3, is now,

$$
\mathcal{M}^{C}\left(k, k^{\prime} ; P\right)=g^{2} \bar{u}\left(p^{\prime}\right)\left[\frac{\not k}{2 p \cdot k}+\frac{\not k^{\prime}}{2 p \cdot k^{\prime}}-\frac{1}{m}\right] u(p) .
$$

This amplitude differs from (23) by an extra term proportional to $\mathrm{m}^{-1}$.

We introduce this amplitude in (22) and replace the massless propagators by Dirac deltas to obtain the discontinuity function:

$$
\begin{aligned}
{\left[\mathcal{M}^{(4)}\right]_{t}=} & -\frac{i g^{4}}{8 \pi^{2}} \int d \Phi \bar{u}_{a}^{\prime}\left[\frac{p_{a} \cdot\left(k^{\prime}-k\right) \not k}{2 p_{a} \cdot k^{\prime} p_{a} \cdot k}\right] u_{a} \bar{u}_{b}^{\prime}\left[\frac{p_{b} \cdot\left(k^{\prime}-k\right) \not k}{2 p_{b} \cdot k^{\prime} p_{b} \cdot k}\right] u_{b} \\
& -\frac{i g^{4}}{8 \pi^{2}} \int d \Phi\left\{\bar{u}_{a}^{\prime} u_{a} \bar{u}_{b}^{\prime} u_{b} \frac{1}{m_{a} m_{b}}-\frac{1}{m_{a}} \bar{u}_{a}^{\prime} u_{a} \bar{u}_{b}^{\prime}\left[\frac{p_{b} \cdot\left(k^{\prime}-k\right)}{2 p_{b} \cdot k p_{b} \cdot k^{\prime}} \not k\right] u_{b}\right. \\
& \left.-\frac{1}{m_{b}} \bar{u}_{a}^{\prime}\left[\frac{p_{a} \cdot\left(k^{\prime}-k\right)}{2 p_{a} \cdot k p_{a} \cdot k^{\prime}} \not k\right] u_{a} \bar{u}_{b}^{\prime} u_{b}\right\} .
\end{aligned}
$$

The first piece is exactly what we had in the last section. We call $\left[\Delta \mathcal{M}^{(4)}\right]_{t}$ the extra added piece that involves the integrations

$$
-\frac{i g^{4}}{8 \pi^{2}} \int d \Phi \bar{u}_{a}^{\prime} u_{a} \bar{u}_{b}^{\prime} u_{b} \frac{1}{m_{a} m_{b}}=-\frac{i g^{4}}{16 \pi m_{a} m_{b}} \bar{u}_{a}^{\prime} u_{a} \bar{u}_{b}^{\prime} u_{b}
$$

and

$$
\begin{aligned}
& \frac{i g^{4}}{8 \pi^{2} m_{a}} \int d \Phi \bar{u}_{a}^{\prime} u_{a} \bar{u}_{b}^{\prime}\left[\frac{p_{b} \cdot\left(k^{\prime}-k\right)}{2 p_{b} \cdot k p_{b} \cdot k^{\prime}} \not k\right] u_{b}+(a \leftrightarrow b) \\
& =\frac{i g^{4}}{8 \pi^{2} m_{a}} \int d \Phi \bar{u}_{a}^{\prime} u_{a} \bar{u}_{b}^{\prime} \not k u_{b} \frac{i \sqrt{t} \xi_{b} m_{b} x_{b}}{\frac{t}{2}\left(\frac{t}{4}+\left(\xi_{b} m_{b} x_{b}\right)^{2}\right)}+(a \leftrightarrow b)
\end{aligned}
$$




$$
\begin{aligned}
& =-\frac{g^{4}}{8 \pi m_{a} \sqrt{t} \xi_{b} m_{b}} \bar{u}_{a}^{\prime} u_{a} \bar{u}_{b}^{\prime} \gamma_{\mu} u_{b}\left\langle\frac{x_{b}}{d_{b}} k^{\mu}\right\rangle+(a \leftrightarrow b) \\
& =\frac{i g^{4}}{16 \pi m_{a} m_{b} \xi_{b}^{2}}\left(1-\tau_{b} \arctan \left(\frac{1}{\tau_{b}}\right)\right) \bar{u}_{a}^{\prime} u_{a} \bar{u}_{b}^{\prime} u_{b}+(a \leftrightarrow b) .
\end{aligned}
$$

The last line in equation (66) is reached by demanding Lorentz covariance to write

$$
\left\langle\frac{x_{b}}{d_{b}} k^{\mu}\right\rangle=a_{b} P_{a}^{\mu}+b_{b} P_{b}^{\mu}+c_{b} Q^{\mu}
$$

by solving for the coefficients as explained in the appendix, and by using the Dirac equation.

Putting things together,

$$
\begin{aligned}
{\left[\Delta \mathcal{M}^{(4)}\right]_{t}=} & \frac{i g^{4}}{16 \pi m_{a} m_{b}} \bar{u}_{a}^{\prime} u_{a} \bar{u}_{b}^{\prime}\left[\frac{1}{\xi_{a}^{2}}\left(1-\tau_{a} \arctan \left(\frac{1}{\tau_{a}}\right)\right)\right. \\
& \left.+\frac{1}{\xi_{b}^{2}}\left(1-\tau_{b} \arctan \left(\frac{1}{\tau_{b}}\right)\right)-1\right] u_{a} u_{b}
\end{aligned}
$$

To leading order in $t$ and $p^{2}$ we have

$$
\left[\Delta \mathcal{M}^{(4)}\right]_{t}=\frac{i g^{4}}{16 \pi m_{a} m_{b}} \bar{u}_{a}^{\prime} u_{a} \bar{u}_{b}^{\prime} u_{b}
$$

In the nonrelativistic limit this contributes the quantity

$$
\Delta V_{n r}^{(4)}=\frac{1}{4 \pi^{2} r} \int_{0}^{\infty} \Delta \rho e^{-\sqrt{t} r} d t=\frac{g^{4}}{64 \pi^{3} r^{3} m_{a} m_{b}}
$$

which exactly cancels contribution (61), i.e.

$$
V_{d e r ; n r}^{(4)}=V_{Y ; n r}^{(4)}+\Delta V_{n r}^{(4)}=0+\mathcal{O}\left(r^{-4}\right)
$$

Hence, to find the form for the potential in the case under scrutiny, we must go to the next order in our series expansions. What we need now is to consistently take into account the previously neglected terms in the spectral density

$$
\rho_{\text {der }}^{(4)} \equiv \frac{\left[\mathcal{M}_{Y}^{(4)}+\Delta \mathcal{M}^{(4)}-\mathcal{M}_{I}\right]_{t}}{2 i} .
$$

So we collect the relevant pieces in (57), (53), and (54) in addition to

$$
[\Delta \mathcal{M}]_{t}^{(4)} \sim \ldots-\frac{i g^{4} \sqrt{t}}{16 m_{a} m_{b}} \bar{u}_{a}^{\prime} \bar{u}_{b}^{\prime}\left(\frac{1}{4 m_{a}}+\frac{1}{4 m_{b}}\right) u_{a} u_{b}
$$


which is the next to leading term in the expansion of (68). The result is

$$
\begin{aligned}
\rho_{d e r}^{(4)} \equiv & \frac{\left[\mathcal{M}_{Y}^{(4)}+\Delta \mathcal{M}-\mathcal{M}_{I}\right]_{t}}{2 i} \\
= & \frac{g^{4} m_{a} m_{b} \sqrt{t}}{8\left(m_{a}+m_{b}\right)} \bar{u}_{a}^{\prime} \bar{u}_{b}^{\prime}\{ \\
& -\frac{3 m_{a}^{4}+5 m_{a}^{3} m_{b}+7 m_{a}^{2} m_{b}^{2}+5 m_{a} m_{b}^{3}+3 m_{b}^{4}}{64 m_{a}^{4} m_{b}^{4}} \\
& +\gamma_{a}^{0} \frac{3 m_{a}^{4}+5 m_{a}^{3} m_{b}-2 m_{a} m_{b}^{3}-3 m_{b}^{4}}{64 m_{a}^{4} m_{b}^{4}} \\
& +\gamma_{b}^{0} \frac{-3 m_{a}^{4}-2 m_{a}^{3} m_{b}+5 m_{a} m_{b}^{3}+3 m_{b}^{4}}{64 m_{a}^{4} m_{b}^{4}} \\
& +\gamma_{a}^{0} \gamma_{b}^{0} \frac{3 m_{a}^{4}+2 m_{a}^{3} m_{b}+7 m_{a}^{2} m_{b}^{2}+2 m_{a} m_{b}^{3}+3 m_{b}^{4}}{64 m_{a}^{4} m_{b}^{4}}
\end{aligned}
$$

+spin-dependent terms of the kind $\left.\gamma_{a} \cdot \gamma_{b}\right\} u_{a} u_{b}$.

This spectral density nonetheless gives a vanishing spin-independent potential in the static approximation, i.e.

$$
V_{d e r ; n r}^{(4)}=0+\mathcal{O}\left(r^{-5}\right)
$$

The first non-vanishing contribution to the spin-independent potential arises from the part in the spectral density which is linear in $t$. Indeed, the explicit form of the spectral density reads:

$$
\begin{aligned}
& \rho^{(4)} \equiv \quad \frac{\left[\mathcal{M}_{Y}^{(4)}+\Delta \mathcal{M}^{(4)}\right]_{t}}{2 i}=\frac{g^{4}}{8 \pi} \frac{1}{240 m_{a}^{4} m_{b}^{4}} \bar{u}_{a}^{\prime} \bar{u}_{b}^{\prime}\{ \\
& 4\left[\left(-3 m_{a}^{4}-2 m_{a}^{3} m_{b}+2 m_{a}^{2} m_{b}^{2}-2 m_{a} m_{b}^{3}-3 m_{b}^{4}\right) p^{2}\right. \\
& \left.+\left(6 m_{a}^{4}+3 m_{a}^{3} m_{b}+4 m_{a}^{2} m_{b}^{2}+3 m_{a} m_{b}^{3}+6 m_{b}^{4}\right) t\right] \\
& +\gamma_{a}^{0} 2\left(m_{a}+m_{b}\right)\left[\left(6 m_{a}^{3}-2 m_{a}^{2} m_{b}+3 m_{a} m_{b}^{2}-4 m_{b}^{3}\right) p^{2}\right. \\
& \left.+\left(-12 m_{a}^{3}+6 m_{a}^{2} m_{b}-4 m_{a} m_{b}^{2}+3 m_{b}^{3}\right) t\right] \\
& +\gamma_{b}^{0} 2\left(m_{a}+m_{b}\right)\left[\left(-4 m_{a}^{3}+3 m_{a}^{2} m_{b}-2 m_{a} m_{b}^{2}+6 m_{b}^{3}\right) p^{2}\right. \\
& \left.+\left(3 m_{a}^{3}-4 m_{a}^{2} m_{b}+6 m_{a} m_{b}^{2}-12 m_{b}^{3}\right) t\right] \\
& +\gamma_{a}^{0} \gamma_{b}^{0}\left[\left(8 m_{a}^{4}+2 m_{a}^{3} m_{b}-12 m_{a}^{2} m_{b}^{2}+2 m_{a} m_{b}^{3}+8 m_{b}^{4}\right) p^{2}\right. \\
& \left.\left.+\left(-6 m_{a}^{4}+2 m_{a}^{3} m_{b}-9 m_{a}^{2} m_{b}^{2}+2 m_{a} m_{b}^{3}-6 m_{b}^{4}\right) t\right]\right\} u_{a} u_{b}
\end{aligned}
$$


where no iterated second order amplitude contributes to this order, and where we picked the term proportional to $t$ in the expansion of (65) and (66).

If we use now

$$
\bar{u}_{a}^{\prime} \gamma_{a}^{0} u_{a} \approx \bar{u}_{a}^{\prime}\left(\mathbf{1}+\mathcal{O}\left(p^{2}\right)\right) u_{a}
$$

and pass to the static limit, we find

$$
\rho_{n r}^{(4)}=\frac{t}{32 m_{a}^{2} m_{b}^{2}} \mathbf{1}_{a} \otimes \mathbf{1}_{b}
$$

which, upon Laplace transformation, leads to

$$
V_{d e r ; n r}^{(4)}=\frac{3 g^{4}}{128 \pi^{3} m_{a}^{2} m_{b}^{2}} \frac{1}{r^{5}}
$$

for the desired spin-independent long range potential. Note that, as opposed to the Yukawa type coupling potential (61), the derivative interaction leads to a repulsive potential.

\section{CONCLUSIONS}

Very light particles can mediate forces extending over distances on the order of their Compton wavelength. If this range is macroscopic, unpolarized bulk matter will only experience the effect of spin-independent interactions. It is a well known fact that the Yukawa potential due to pseudoscalar exchange depends on spin and as a consequence no coherent effects do arise on a macroscopic scale, unless of course our sample is polarized [5]. However, residual (Van der Waals type) forces may arise between macroscopic bodies in the case of pseudoscalar mediated interactions, due to the exchange of two quanta "at the same time". The double helicity flip involved eventually makes the resulting effective potential spin-independent [7].

In the preceding sections we have established, with the help of the formalism developed by Feinberg and Sucher, the large distance behavior of such residual forces, i.e. those associated to double pseudoscalar exchange. We have considered two different basic couplings of those scalars to matter fermions. On the one hand we took the ordinary Yukawa coupling (e.g., 
this is the way the Higgs particle couples to fermions) and on the other we considered the derivative coupling (e.g., the axion-fermion interaction). Both couplings reduce to the same spin-flip interaction in the static non-relativistic limit. And both interactions also produce identical one-particle exchange (spin-dependent) potentials. In spite of this fact, we have explicitly shown that the spin-independent two-particle exchange potential is substantially different in both cases. Indeed, for Yukawa coupling we derive a $1 / r^{3}$ attractive long distance behavior whereas for the derivative coupling the potential, that is now repulsive, falls off as $1 / r^{5}$. Since, as emphasized, double exchange will lead to residual macroscopic effects, these

effects will be quite different in both cases. So, we have found still another instance where the interaction of pseudoscalars to fermions can be discriminated. Other places are, soft pion emission in proton-proton scattering or axion bremsstrahlung in a supernova core [4, 15].

Of course, the effects just reported are extremely small for the light scalars presently contemplated in particle physics, such as the axion, and thus their experimental detection is beyond reach of present technology. However, there is much activity and interest on the experimental front and experiments are designed and performed that explore the subcentimeter and sub-millimeter regime with an ever increasing sensitivity [16],17]. And, on the theoretical side, the completion of the Particle Physics Paradigm may still require new superlight scalar particles to exist.

\section{ACKNOWLEDGMENTS}

Work partially supported by the CICYT Research Project AEN95-0882. F.F. acknowledges the CIRIT for financial support.

\section{APPENDIX:}

The coefficients $a_{i}$

The coefficients in the tensor decomposition (33) read: 


$$
\begin{aligned}
& a_{1}=\frac{t\left(I_{1}-2 I_{5}-I_{6}+4 y I_{4}-y^{2} I_{1}-y^{2} I_{6}\right)}{16 m_{a}^{2} \xi_{a}^{2}\left(1-y^{2}\right)^{2}} \\
& a_{2}=\frac{t\left(I_{1}-I_{5}-2 I_{6}+4 y I_{4}-y^{2} I_{1}-y^{2} I_{5}\right)}{16 m_{b}^{2} \xi_{b}^{2}\left(1-y^{2}\right)^{2}} \\
& a_{3}=\frac{t\left(-I_{4}-y I_{1}+2 y I_{5}+2 y I_{6}-3 y^{2} I_{4}+y^{3} I_{1}\right)}{16 b\left(1-y^{2}\right)^{2}} \\
& a_{4}=-\frac{t\left(I_{1}-I_{5}-I_{6}+2 y I_{4}-y^{2} I_{1}\right)}{4\left(1-y^{2}\right)}
\end{aligned}
$$

with $y \equiv \hat{\mathbf{p}} \cdot \hat{\mathbf{p}}^{\prime}$, in terms of various angular integrals in the set

$$
\begin{array}{lll}
I_{0} \equiv\left\langle\frac{1}{d_{a} d_{b}}\right\rangle & I_{1} \equiv\left\langle\frac{x_{a} x_{b}}{d_{a} d_{b}}\right\rangle & I_{2} \equiv\left\langle\frac{x_{a}^{2}}{d_{a} d_{b}}\right\rangle \\
I_{3} \equiv\left\langle\frac{x_{b}^{2}}{d_{a} d_{b}}\right\rangle & I_{4} \equiv\left\langle\frac{x_{a}^{2} x_{b}^{2}}{d_{a} d_{b}}\right\rangle & I_{5} \equiv\left\langle\frac{x_{a}^{3} x_{b}}{d_{a} d_{b}}\right\rangle \\
I_{6} \equiv\left\langle\frac{x_{a} x_{b}^{3}}{d_{a} d_{b}}\right\rangle .
\end{array}
$$

These results are obtained after repeated contraction of (33) with the independent momenta in our problem and after solving the resulting algebraic system of equations.

The angular integrals $I_{i}$ are given next. The explicit calculation of $I_{i}$ for $i<5$ is given in 13 I3 $I_{5}$ can be found in 14 and $I_{6}$ follows trivially from $I_{5}$ by interchanging $a$ and $b$. For the first two integrals from the set (A2) one gets

$$
I_{0}=\frac{F_{+}+\pi N_{+}^{-1}}{2 \tau_{a} \tau_{b}} \quad I_{1}=\frac{F_{-}+\pi N_{+}^{-1}}{2}
$$

where we have defined,

$$
\begin{aligned}
& F_{ \pm}= \pm N_{-}^{-1} \arctan \left(\frac{N_{-}}{D_{+}}\right)-N_{+}^{-1} \arctan \left(\frac{N_{+}}{D_{-}}\right) \\
& N_{+}=-\frac{i p s^{1 / 2}}{b} \\
& N_{-}=-\frac{i \sqrt{p^{2} s+b y t}}{b} \\
& D_{ \pm}=y \pm \tau_{a} \tau_{b}
\end{aligned}
$$

and the rest is given in terms of $I_{0}$ and $I_{1}$ by

$$
I_{2}=\frac{1}{\tau_{b}} \arctan \left(\frac{1}{\tau_{b}}\right)-\tau_{a}^{2} I_{0}
$$




$$
\begin{aligned}
& I_{3}=\frac{1}{\tau_{a}} \arctan \left(\frac{1}{\tau_{a}}\right)-\tau_{b}^{2} I_{0} \\
& I_{4}=1-\tau_{a} \arctan \left(\frac{1}{\tau_{a}}\right)-\tau_{b} \arctan \left(\frac{1}{\tau_{b}}\right)+\tau_{a}^{2} \tau_{b}^{2} I_{0} \\
& I_{5}=y\left(1-\tau_{b} \arctan \left(\frac{1}{\tau_{b}}\right)\right)-\tau_{a}^{2} I_{1} \\
& I_{6}=y\left(1-\tau_{a} \arctan \left(\frac{1}{\tau_{a}}\right)\right)-\tau_{b}^{2} I_{1} .
\end{aligned}
$$

\section{Angular integrals entering the iterated amplitude}

We start with (42). Its discontinuity $[L]_{t}$ has been obtained in [13]:

$$
[\mathcal{L}]_{t}=i\left[\frac{\pi}{l \sqrt{t}} \frac{1}{\sqrt{\left(l_{+}^{2}-l^{2}\right)\left(l^{2}-l_{-}^{2}\right)}}\right] \Theta\left(l_{+}^{2}-l^{2}\right) \Theta\left(l^{2}-l_{-}^{2}\right)
$$

with

$$
l_{ \pm}^{2}=\frac{a^{\prime} \pm b^{\prime}}{2} \quad a^{\prime}=2 p^{2}+t, \quad b^{\prime}=\sqrt{t\left(4 p^{2}+t\right)}
$$

For $p^{2}=l^{2}$, simplifies to

$$
\left.[\mathcal{L}]_{t}\right|_{p^{2}=l^{2}}=\frac{i \pi}{p^{2} t}
$$

We turn now to (43). It has been calculated in 14. It is symmetric in $\mathbf{p}$ and $\mathbf{p}^{\prime}$. Hence it can be cast in the form

$$
\mathcal{V}=v \mathbf{P} \quad \text { with } \quad \mathbf{P} \equiv \mathbf{p}+\mathbf{p}^{\prime}
$$

where $v$ is given by

$$
v=\frac{1}{P^{2}}\left(\left(p^{2}+l^{2}\right) \mathcal{L}-\frac{1}{4 p l} \ln \left[\frac{(p+l)^{2}}{(p-l)^{2}}\right]\right) .
$$

The associated discontinuity is then

$$
[\mathcal{V}]_{t}=[v]_{t} \mathbf{P}=\frac{p^{2}+l^{2}}{4 p^{2}+t}[\mathcal{L}]_{t} \mathbf{P}
$$

Finally let us discuss (44). This integral admits the general decomposition 


$$
\Upsilon_{i j}=a P_{i} P_{j}+b Q_{i} Q_{j}+c \delta_{i j}
$$

in terms of the vectors $\mathbf{P}$, defined above, and $\mathbf{Q} \equiv \mathbf{p}-\mathbf{p}^{\prime}$. Clearly, we can write

$$
\begin{gathered}
P_{i} P_{j} \Upsilon^{i j}=a\left(P^{2}\right)^{2}+c P^{2} \\
Q_{i} Q_{j} \Upsilon^{i j}=b\left(Q^{2}\right)^{2}+c Q^{2} \\
\delta_{i j} \Upsilon^{i j}=a P^{2}+b Q^{2}+3 c
\end{gathered}
$$

(we used: $\mathbf{P} \cdot \mathbf{Q}=0$ ). The scalar integrals on the left are, respectively

$$
\begin{aligned}
P_{i} P_{j} \Upsilon^{i j}= & \int \frac{d \Omega}{4 \pi} \frac{(\mathbf{P} \cdot \mathbf{l})^{2}}{\left(\mathbf{q}^{\prime 2}\right)\left(\mathbf{q}^{2}\right)} \\
= & \left(p^{2}+l^{2}\right) \mathcal{L}-\frac{p^{2}+l^{2}}{2 p l} \ln \left[\frac{(p+l)^{2}}{(p-l)^{2}}\right] \\
& +\frac{4 p^{2}+t}{4 p^{2}}+\frac{p^{2}+l^{2}}{16 p^{3} l} t \ln \left[\frac{(p+l)^{2}}{(p-l)^{2}}\right] \\
Q_{i} Q_{j} \Upsilon^{i j}= & \frac{1}{4}\left(-2+\frac{2 p^{2}+t}{p^{2}}+\frac{p^{2}+l^{2}}{4 p^{3} l} t \ln \left[\frac{(p+l)^{2}}{(p-l)^{2}}\right]\right) \\
\delta_{i j} \Upsilon^{i j}= & l^{2} \mathcal{L} .
\end{aligned}
$$

Now the three equations can be solved for $a, b$, and $c$. Recall that we only need the discontinuity of (44), i.e. the discontinuities of $a, b$, and $c$. They are,

$$
\begin{aligned}
{[a]_{t} } & =\frac{1}{4 p^{2}+t}\left(2 \frac{\left(p^{2}+l^{2}\right)^{2}}{4 p^{2}+t}-l^{2}\right)[\mathcal{L}]_{t} \\
{[b]_{t} } & =\frac{[c]_{t}}{t}[\mathcal{L}]_{t} \\
{[b]_{t} } & =\left(l^{2}-\frac{\left(p^{2}+l^{2}\right)^{2}}{4 p^{2}+t}\right)[\mathcal{L}]_{t} .
\end{aligned}
$$

\section{Integrals $I_{c 1}$ and $I_{c 2}$}

Here we display the explicit solutions of (51) and (52) for $n=0,2,4,6$ :

$$
\begin{aligned}
& I_{c 1}(0)=0 \\
& I_{c 1}(2)=-\pi \\
& I_{c 1}(4)=-\frac{\pi}{2}\left(4 p^{2}+t\right) \\
& I_{c 1}(6)=-\frac{\pi}{8}\left(\left(4 p^{2}+t\right)^{2}+2\left(2 p^{2}+t\right)^{2}\right)
\end{aligned}
$$


and,

$$
\begin{aligned}
& I_{c 2}(0)=\pi \\
& I_{c 2}(2)=\frac{\pi}{2}\left(2 p^{2}+t\right) \\
& I_{c 2}(4)=\frac{\pi}{8}\left(8 p^{4}+12 p^{2} t+3 t^{2}\right) \\
& I_{c 2}(6)=\frac{\pi}{16}\left(16 p^{6}+48 p^{4} t+30 p^{2} t^{2}+5 t^{3}\right) .
\end{aligned}
$$




\section{REFERENCES}

[1] G.B. Gelmini, S. Nussinov and T. Yanagida, Nucl. Phys. B219, 31 (1983).

[2] I. Antoniadis, S. Dimopoulos and G. Dvali, Nucl. Phys. B516, 70 (1998).

[3] F. Wilczek, Phys. Rev. Lett. 49, 1549 (1982).

[4] G.G. Raffelt, Phys. Rep. 198 (1990).

[5] J. E. Moody and F. Wilczek, Phys. Rev. D30, 130 (1984); F. Wilczek, hep-ph/9802400.

[6] E. Fischbach and C. Talmadge,in Proceedings of the XXIIIrd Rencontre de Moriond, O. Fackler i J. Tran Thanh Van eds., (1988).

[7] J.A. Grifols and S. Tortosa Phys. Lett. B328, 98 (1994).

[8] G. Feinberg, J. Sucher and C.K. Au, Phys. Rep. 180, 85 (1989).

[9] G. Feinberg and J. Sucher, in Long-Range Casimir Forces: Theory and Recent Experiments in Atomic Systems, edited by Frank S. Levin and David A. Micha (Plenum Press, New York, 1993).

[10] F. Ferrer, Diploma Thesis, U.A.B. internal Report.

[11] S. Tortosa, Diploma Thesis, U.A.B. internal Report.

[12] J. Sucher, Phys. Rev. Lett. 55, 1023 (1985); J. Sucher, hep-ph/9412388.

[13] G. Feinberg and J. Sucher, Phys. Rev. D38, 3763 (1988).

[14] G. Feinberg and J. Sucher, Phys. Rev. D45, 2493 (1992).

[15] M.S. Turner, H.S. Hang and G. Steigman, Phys. Rev. D40, 299 (1989); G. Raffelt and D. Seckel, Phys. Rev. Lett. 60, 1793 (1988).

[16] J. C. Long, H. W. Chan and J. C. Price, hep-ph/9805217.

[17] M. Bordag, B. Geyer, G. L. Klimchitskaya and V. M. Mostepanenko, hep-ph/9804223. 


\section{Figure Captions}

FIG. 1 Lowest order scattering amplitude. Single pseudoescalar exchange.

FIG. 2 Diagrams contributing to the $\mathcal{O}\left(g^{4}\right)$ terms of the potential.

FIG. 3 Compton scattering amplitude diagrams. 


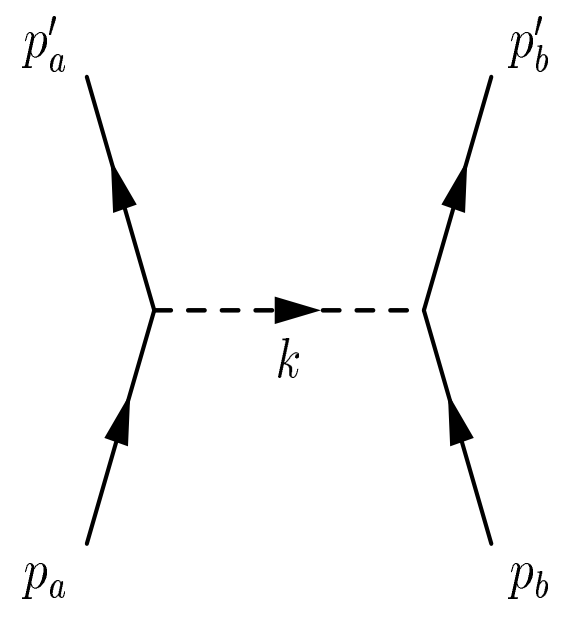

FIG. 1.

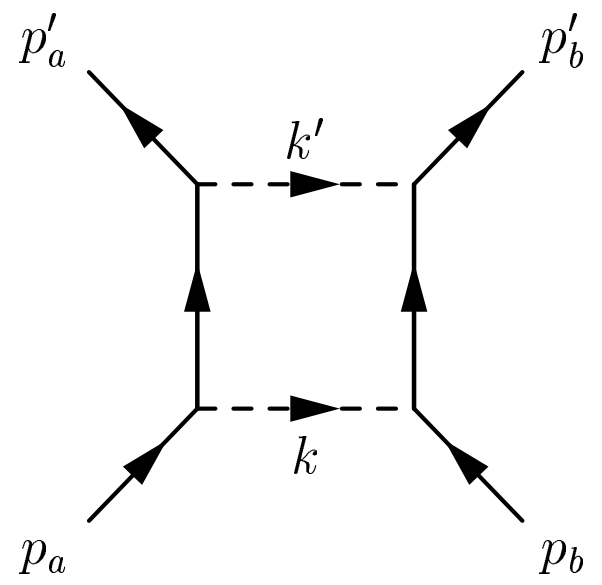

(a)

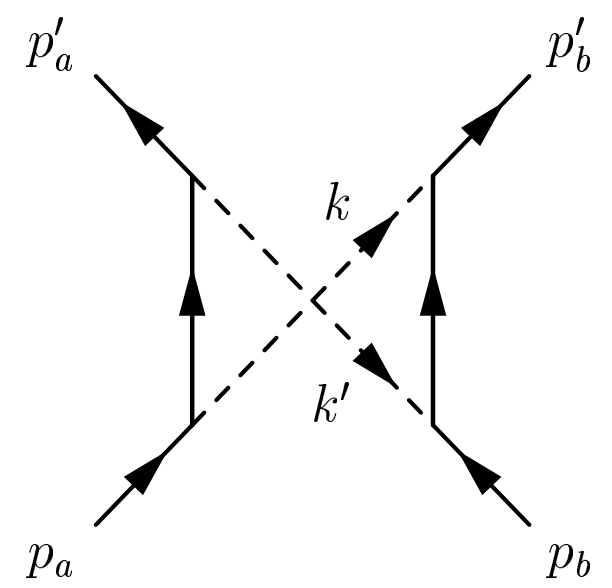

(b)

FIG. 2. 


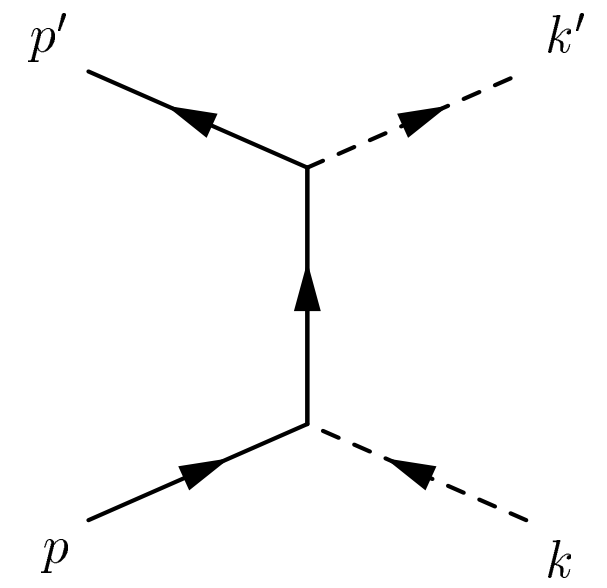

(a)

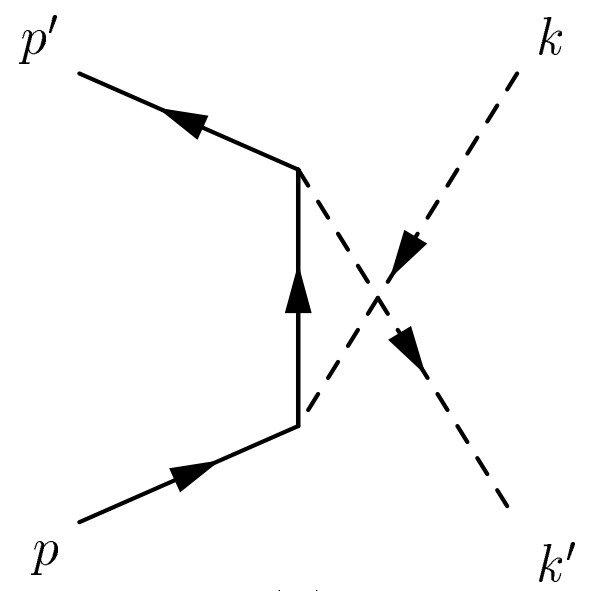

(b)

FIG. 3. 